\title{
Is $\xi$ Boo AB a Young or an Evolved System? A Model Atmosphere Analysis
}

\author{
I.S. Savanov \\ Crimean Astrophysical Observatory, Nauchny, 334413 Crimea, USSR
}

\begin{abstract}
We perform a model atmosphere analysis of $\xi$ Boo A, and make estimates for the secondary component. The abundances of 25 elements for $\xi$ Boo $\mathrm{A}$ are obtained with the parameters: $T_{\text {eff }}=(5300 \pm 100) \mathrm{K}, \log g=4.1 \pm 0.2, \xi_{\mathrm{t}}=0.5 \mathrm{~km} / \mathrm{s}$. The atmosphere of $\xi \mathrm{Boo} \mathrm{A}$ is deficient in $\mathrm{Y}$ and $\mathrm{Zr}$, but overabundant in $\mathrm{Ba}$ and rare earth elements. We confirm the high $\mathrm{Li}$ abundance in $\xi$ Boo $\mathrm{A}(\log A(\mathrm{Li})=2.1)$, while $\mathrm{Li}$ is strongly underabundant in the secondary relative to the Sun. From comparison with evolutionary calculations we find that $\xi$ Boo A has mass $0.95 M_{\odot}$, radius $1.4 R_{\odot}$; we also determine a rotational velocity $v \sin \dot{\xi}=8 \mathrm{~km} / \mathrm{s}$. We conclude that, in spite of its relatively high rotation rate and high chromospheric activity, it is already evolved from the main-sequence.
\end{abstract}

The components of the $\xi$ Boo AB (HD 131156) have been the objects of many investigations since 1965 when Herbig noted that the $\mathrm{Li}$ abundance in the atmosphere of the primary is one of the highest among the $G$ type stars. This star has strong and variable $\mathrm{Ca}$ II and ultraviolet emission line fluxes (Baliunas et al., 1985; Noyes et al., 1984). Measurements of magnetic field on $\xi$ Boo A have indicated the possibility of its variability (e.g. Marcy, 1984). From observations of Ca II $\mathrm{K}$ emission and linear polarization, Saar et al. (1988) constructed a map of the stellar active regions.

We performed our model atmosphere analysis on the basis of high dispersion observational data published by Boyarchuk and Eglitis (1983) and additionally obtained high S/N spectra with the CCD-camera in the coudé focus of the $2.6 \mathrm{~m}$ Shajn reflector in Crimean Astrophysical Observatory. Models of atmospheres by Bell et al. (1975) were used in the analysis. The estimates of the effective temperature and gravity were made on the basis of the $T_{\text {eff }}-\log g$ diagram (Fig. 1). The statistical dependence $\log T_{\text {eff }}=3.908-0.234(B-V)$ was applied according to Noyes et al. (1984). The microturbulence was determined from the lines of $\mathrm{Fe} I$.

Abundances of 25 elements were obtained with the following parameters for the A component: $T_{\text {eff }}=(5300 \pm 100) \mathrm{K}, \log g=4.1 \pm 0.2$ and $\xi_{\mathfrak{t}}=(0.5 \pm 0.5) \mathrm{km} / \mathrm{s}$. Figure 2 shows the results of our calculations. Besides $\mathrm{Li}, \mathrm{Ba}$ and heavier elements also show overabundance. The mean value of the overabundance of the rare earth elements is equal to $0.77 \mathrm{dex} . \mathrm{Y}$ and $\mathrm{Zr}$, on the contrary, are underabundant.

According to the measurements of the equivalent width of the $\operatorname{Li}$ I $\lambda 6707 \AA$ blend from the CCD spectrogram obtained on June 27, 1986, the $\mathrm{Li}$ abundance is 2.1. Our value of the ratio $[\mathrm{Li} / \mathrm{Ca}]$ is 1.05 and is in agreement with the estimate 


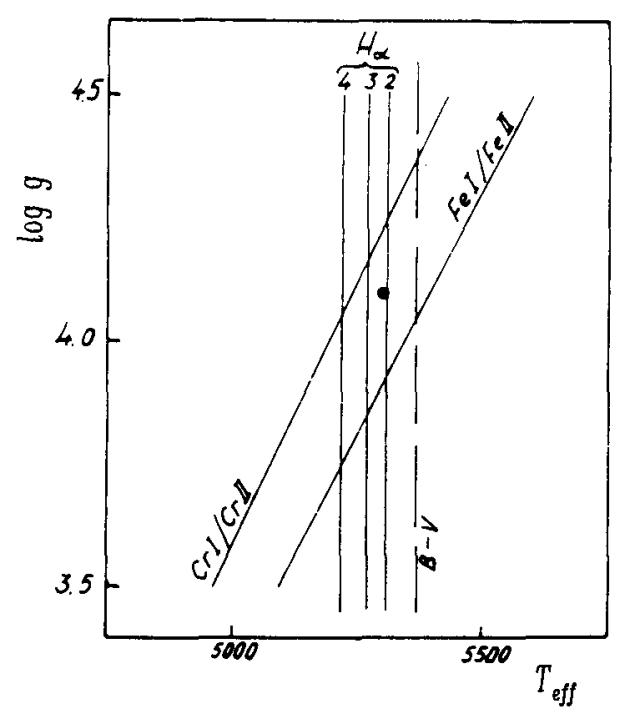

Fig. 1. $\log T_{\text {eff }}-\log g$ diagram. The circle refers to the adopted model.

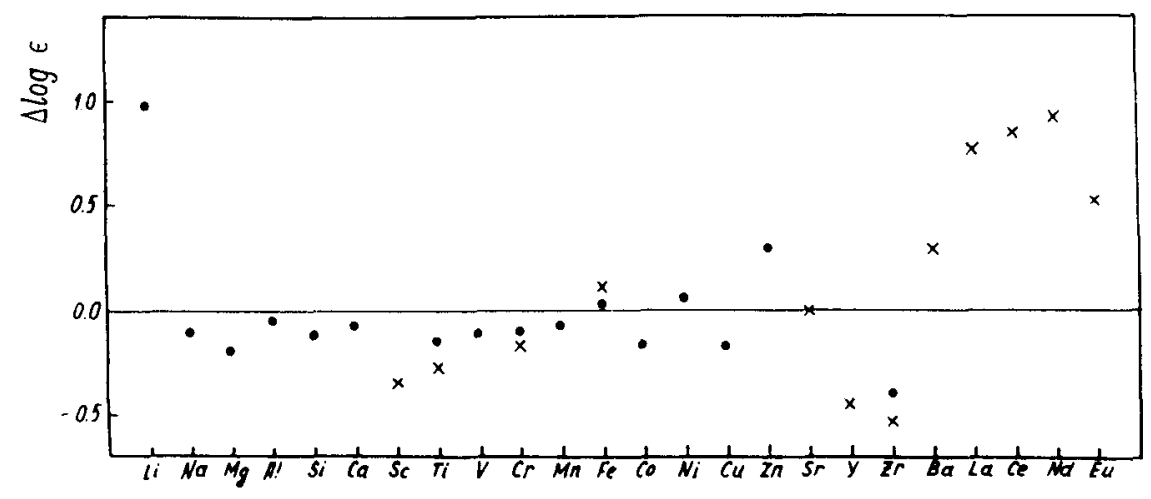

Fig. 2. Comparison between the chemical composition of the $\xi$ Boo $A$ and solar atmosphere. Open circles represent abundances obtained from lines of neutral elements and crosses represent the results from the ions.

by Herbig (1965) who obtained $[\mathrm{Li} / \mathrm{Ca}]=1.39$. We could not find the lithium line in the spectra of the secondary. We conclude that equivalent width of the lithium line in the spectrum of $\xi$ Boo B is less than $4 \mathrm{~m} \AA$. Assuming for the component B the atmospheric parameters $T_{\text {eff }}=4500 \mathrm{~K}, \log g=4.5$ and $\xi_{\mathrm{t}}=0.5 \mathrm{~km} / \mathrm{s}$, we found that difference in the $\mathrm{Li}$ abundance in the atmospheres of the components is more than 2.5 dex.

Using the evolutionary calculations by Mengel et al. (1979) we found the mass, radius and luminosity of $\xi$ Boo $\mathrm{A}: M / M_{\odot}=0.95, R / R_{\odot}=1.4$ and $L / L_{\odot}=1.5$ (Fig.3). The precision of the mass estimate is connected with the uncertainties 
of the $\log g$ determination. Our spectroscopic mass is slightly larger than that given by the equation $\log \left(M / M_{\odot}\right)=0.38-0.42(B-V)$ (Noyes et al., 1974). According to this equation the masses of the components are 0.91 and $0.61 M_{\odot}$. These values are in agreement with the total mass of the system $(1.58 \pm 0.19) M_{\odot}$ (Wielen, 1962).

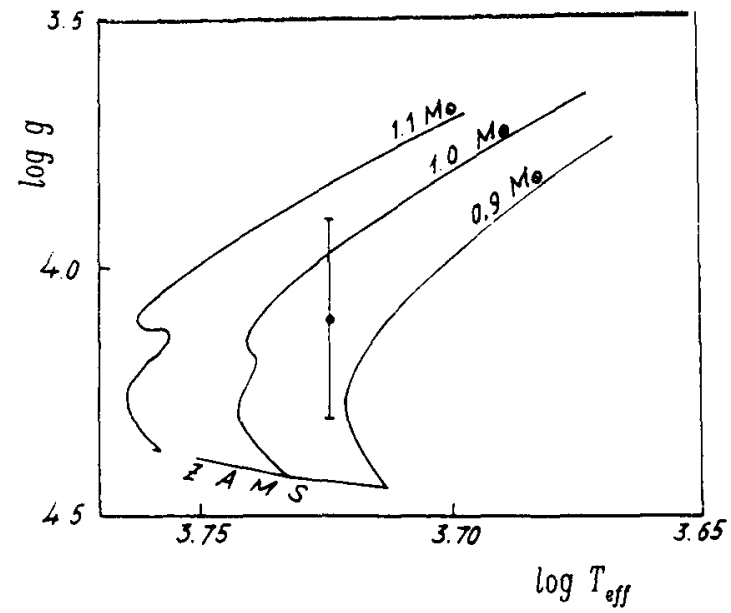

Fig. 3. Evolutionary tracks for the stars with $0.9,1.0$ and $1.1 M_{\odot}$. The circle refers to the position of $\xi$ Boo A.

From the comparison of line profile calculations of Ca I $\lambda 6717 \AA$ with that observed in the spectrum of $\xi$ Boo A we obtain $v \sin i=(8 \pm 2) \mathrm{km} / \mathrm{s}$. Assuming $R=1.4 R_{\odot}$ and that the period of the variations in equivalent widths and spectral line asymmetries is $P=6.43 \mathrm{~d}$ (Toner and Gray, 1988) we conclude that the inclination of the rotational axis is about $45^{\circ}$ and is close to the inclination of the orbit of the whole system, which according to Wielen (1962) is $40^{\circ}$.

On the basis of our results we have to conclude that $\xi$ Boo $A$ has already evolved from the main sequence and is not a young main sequence star. This result seems not to be in accordance with the high chromospheric activity and relatively high rotation rate of the star.

\section{References}

Baliunas, S.L. et al.: 1985, Astrophys. J. 294, 310

Bell, R.A., Eriksson, K., Gustafsson, B., Nordlund, A.: 1976, Astron. Astrophys. Suppl. 23, 37

Boyarchuk, A.A., Eglitis, I.: 1983, Bull. Crimean Astrophys. Obs. 67, 13

Herbig, G.H.: 1965, Astrophys. J. 141, 588

Marcy, G.W.: 1984, Astrophys. J. 275, 286

Mengel, J.D.: 1979, Astrophys. J. Suppl. Ser. 40, 733

Noyes, R.W. et al.: 1984, Astrophys. J. 279, 763

Saar, S.H., Huovelin, J., Tuominen, I.: 1988, Astrophys. J. 329, 882

Toner, C.G., Gray, D.F.: 1988, Astrophys. J. 334, 1008

Wielen, R.: 1962, Astron. J. 67, 599 